\title{
EXTENSIONS OF LOCALLY COMPACT ABELIAN GROUPS. I
}

\author{
BY \\ RONALD O. FULP AND PHILLIP A. GRIFFITH( ${ }^{(1)}$
}

\begin{abstract}
This paper is concerned with the development of a (discrete) groupvalued functor Ext defined on $\mathscr{L} \times \mathscr{L}$ where $\mathscr{L}$ is the category of locally compact abelian groups such that, for $A$ and $B$ groups in $\mathscr{L}$, Ext $(A, B)$ is the group of all extensions of $B$ by $A$. Topological versions of homological lemmas are proven to facilitate the proof of the existence of such a functor. Various properties of Ext are obtained which include the usual long exact sequence which connects Hom to Ext. Along the way some applications are obtained one of which yields a slight improvement of one of the Noether isomorphism theorems. Also the injectives and projectives of the category of locally compact abelian totally disconnected groups are obtained. They are found to be necessarily discrete and hence are the same as the injectives and projectives of the category of discrete abelian groups. Finally we obtain the structure of those connected groups $C$ of $\mathscr{L}$ which are direct summands of every $G$ in $\mathscr{L}$ which contains $C$ as a component.
\end{abstract}

This paper is concerned with homological algebra in the category of locally compact abelian groups and with certain of its applications. In the first part of the paper we lay down the necessary homological framework for the work which is to follow. This framework provides for the development of a functor Ext which generalizes the usual functor Ext as is defined in (discrete) abelian group theory and also generalizes the functor Ext discussed by Moskowitz [10]. We show that Ext has many of the properties as does the ordinary Ext functor whose arguments are discrete groups.

The second part of the paper (roughly from $\S 3$ on) deals with some applications. In particular, we completely determine the structure of those connected locally compact abelian groups $G$ having the property that whenever $H$ is locally compact abelian and $H$ has $G$ as its component, then $G$ is a direct summand of $H$. We actually prove a much better theorem (Theorem 5.2). Other theorems of interest are obtained along the way. For example, the injectives and projectives of the

Presented to the Society, August 30, 1968 and November 9, 1968; received by the editors January 25, 1968 and, in revised form, August 26, 1968 and March 20, 1970.

AMS 1968 subject classifications. Primary 2220; Secondary 2210.

Key words and phrases. Locally compact abelian groups, topological group extensions, homological-topological lemmas, extension functor, Noether theorem, totally disconnected projectives, totally disconnected injectives.

$\left.{ }^{1}\right)$ The research of the first author was supported in part by NASA Grant NGR-44-005037. The second author also wishes to acknowledge support by the National Science Foundation Grant GP-8750.

Copyright (C) 1971, American Mathematical Society 
category of totally disconnected locally compact abelian groups are obtained. Also slight improvement of one of the Noether isomorphism theorems is made.

Various papers deal with the general subject under investigation here. Some results on lower-dimensional cohomology groups by Moore [8] and [9] bear on our subject. The older papers of Nagao and Calibi are referenced in Moore's paper and various extensions of Moore's work appear in more recent papers. The paper [6] by Hofmann and Mostert should also be mentioned.

Actually our work was inspired by the paper [10] of Moskowitz which in turn appears to follow the lead of the two papers [4] and [5] by Hochschild on extensions of Lie groups. Thus we shall assume some familiarity with the paper [10] and with basic facts from the theory of topological groups as are set forth in Pontrjagin [11] or Hewitt and Ross [3]. Moreover our development of the Ext functor requires a rather detailed knowledge of the corresponding collection of ideas in the theory of discrete abelian groups as is exposed in Chapter 3 of the book [7] by Mac Lane.

The category with which we deal is the category of locally compact abelian groups with continuous homomorphisms as morphisms. We shall denote this category by $\mathscr{L}$. Actually most of our results deal only with those morphisms of $\mathscr{L}$ which are open onto their image. Such a morphism will be called a proper morphism. The class of all proper morphisms of $\mathscr{L}$ is not a subcategory of $\mathscr{L}$, but it does have several nice properties which we outline below.

(1) If $\phi: A \rightarrow B$ is a proper morphism of $\mathscr{L}$ then $\operatorname{Im} \phi=\phi(A)$ is a closed subgroup of $B$.

(2) A proper morphism of $\mathscr{L}$ is monic in $\mathscr{L}$ if and only if it is a monomorphism.

(3) A proper morphism of $\mathscr{L}$ is epic in $\mathscr{L}$ if and only if it is an epimorphism.

(4) If $\varphi: A \rightarrow B$ and $\theta: B \rightarrow C$ are proper morphisms of $\mathscr{L}$, then $\theta \varphi$ is a proper morphism if either $\varphi$ is epic or $\theta$ is monic.

We now lay down some notation and terminology to be used throughout the paper. If $\varphi: \mathrm{A} \rightarrow B$ and $\theta: B \rightarrow C$ are morphisms in $\mathscr{L}$, then the sequence $A \stackrel{\varphi}{\rightarrow} B \stackrel{\theta}{\rightarrow} C$ is exact at $B$ if and only if $\operatorname{Im} \varphi=\operatorname{ker} \theta$, i.e. if and only if $\varphi(A)=$ $\theta^{-1}(0)$. A longer sequence of morphisms of $\mathscr{L}$

$$
A_{1} \longrightarrow A_{2} \longrightarrow \cdots \longrightarrow A_{n}
$$

is exact if and only if for each $2 \leqq i \leqq n-1$, the sequence $A_{i-1} \rightarrow A_{i} \rightarrow A_{i+1}$ is exact at $A_{i}$. A sequence $A \stackrel{\varphi}{\rightarrow} B \stackrel{\theta}{\rightarrow} C$ is short exact if and only if it is exact, $\varphi$ is a monomorphism, and $\theta$ is an epimorphism. In general, if $\varphi: A \rightarrow B$ is a morphism of $\mathscr{L}$, we write $A \stackrel{\varphi}{\longrightarrow} B$ whenever $\varphi$ is a monomorphism and $A \stackrel{\varphi}{\rightarrow} B$ whenever $\varphi$ is an epimorphism. Any undefined homological notation or terminology is taken from Mac Lane [7].

An exact sequence of morphisms of

$$
A_{1} \stackrel{\varphi_{1}}{\longrightarrow} A_{2} \stackrel{\varphi_{2}}{\longrightarrow} \cdots \stackrel{\varphi_{n-1}}{\longrightarrow} A_{n}
$$


is said to be proper exact if and only if $\varphi_{i}$ is proper for each $i$. More generally, if $D$ is a diagram with morphisms in $\mathscr{L}$, then $D$ is a proper diagram if and only if each morphism of $D$ is a proper morphism. Thus we speak of proper pullbacks, proper pushouts, etc.

Throughout the paper we repeatedly use the duality theory of locally compact abelian groups. If $G$ is a locally compact abelian group, then the dual of $G$, denoted $G^{\wedge}$, is the topological group of all continuous characters of $G$. If $\varphi: G \rightarrow H$ is a morphism in $\mathscr{L}$, then the map $\varphi^{\wedge}: H^{\wedge} \succ G^{\wedge}$ defined by $\varphi^{\wedge}(\chi)=\chi \circ \varphi$ is also a morphism of $\mathscr{L}$ and will be called the dual of $\varphi$. If $E: A \stackrel{\varphi}{\rightarrow} B \stackrel{\theta}{\rightarrow} C$ is a short exact sequence in $\mathscr{L}$, then the dual of $E$, denoted $E^{\wedge}$, is the short exact sequence $C^{\wedge} \stackrel{\theta^{\wedge}}{\longrightarrow} B^{\wedge} \stackrel{\varphi^{\wedge}}{\rightarrow} A^{\wedge}$. In general, if $D$ is a diagram of $\mathscr{L}$, then the dual of $D$ is the diagram obtained by taking duals of each of the morphisms of $D$.

The additive groups of real numbers, rational numbers, integers, and real numbers modulo 1 will be denoted by $R, Q, Z$, and $T$, respectively. If $A$ is a subset of a topological space, $A^{-}$will denote the closure of $A$. If $G$ is a topological group, $G_{0}$ will denote the identity component of $G$.

2. Homological algebra in $\mathscr{L}$. Our first result in this section establishes a criterion for determining proper morphisms in $\mathscr{L}$ and is the foundation upon which our discussion of homological algebra in $\mathscr{L}$ rests.

THEOREM 2.1. A morphism $\psi$ in $\mathscr{L}$ is proper if and only if there are proper morphisms $\alpha$ and $\beta$ in $\mathscr{L}$ such that

(i) $\psi \alpha$ and $\beta \psi$ are defined and are proper,

(ii) $\operatorname{Im} \psi \alpha=\operatorname{ker} \beta$.

Proof. First suppose that $\psi$ is a morphism in $\mathscr{L}$ and that there are proper morphisms $\alpha$ and $\beta$ satisfying (i) and (ii) above. We show that $\psi$ is open onto its range. Observe that (i) and (ii) yield the following commutative diagram

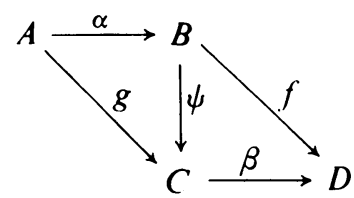

where $\alpha, \beta, f$ and $g$ are proper and $\operatorname{Im} g=\operatorname{ker} \beta$. By Proposition 2.2 of [10], $\operatorname{Im} \alpha$, $\operatorname{Im} \beta, \operatorname{Im} f$ and $\operatorname{Im} g$ are closed in their respective containing groups. We show that $\psi(U)$ has nonvoid interior in $\operatorname{Im} \psi$ and hence that $\psi$ is proper. Choose $U_{1}$ a neighborhood of zero in $B$ such that $U_{1}+U_{1} \subseteq U$. Since $f, g$ and $\alpha$ are proper, there are neighborhoods $S_{1}$ and $W_{1}$ in $D$ and $C$, respectively, such that $S_{1} \cap \operatorname{Im} f=$ $f\left(U_{1}\right)$ and $W_{1} \cap \operatorname{Im} g=g \alpha^{-1}\left(U_{1} \cap \operatorname{Im} \alpha\right)$. Noting that $W_{1} \cap \beta^{-1}\left(S_{1}\right)$ is a neighborhood of zero in $C$, we choose a neighborhood $V_{1}$ of zero such that $V_{1}+\left(-V_{1}\right) \subseteq$ $W_{1} \cap \beta^{-1}\left(S_{1}\right)$. Since $\psi$ is continuous, $U_{2}=U_{1} \cap \psi^{-1}\left(V_{1}\right)$ is a neighborhood of zero in $B$. As before, there are neighborhoods $S_{2}$ and $W_{2}$ in $D$ and $C$, respectively, 
such that $S_{2} \subseteq S_{1}$ and $W_{2} \subseteq W_{1}$ and such that $S_{2} \cap \operatorname{Im} f=f\left(U_{2}\right)$ and $W_{2} \cap \operatorname{Im} g=$ $g \alpha^{-1}\left(U_{2} \cap \operatorname{Im} \alpha\right)$. It follows that $V_{2}=V_{1} \cap W_{2} \cap \beta^{-1}\left(S_{2}\right)$ is a neighborhood of zero in $C$. Let $\psi(b) \in V_{2}$. Then $\beta \psi(b)=f(b) \in S_{2} \cap \operatorname{Im} f=f\left(U_{2}\right)$ and hence $\beta \psi(b)=$ $f\left(u_{2}\right)=\beta \psi\left(u_{2}\right)$ for some $u_{2} \in U_{2}$. Observing that $\psi\left(u_{2}\right) \in V_{1}$, we have that $\psi(b)-$ $\psi\left(u_{2}\right) \in\left[V_{1}+\left(-V_{1}\right)\right] \cap \operatorname{ker} \beta \subseteq W_{1} \cap \operatorname{Im} g=g \alpha^{-1}\left(U_{1} \cap \operatorname{Im} \alpha\right)$. Therefore $\psi(b)-$ $\psi\left(u_{2}\right)=g(a)$ where $\alpha(a) \in U_{1}$. It follows that $u_{2}+\alpha(a) \in U_{1}+U_{1} \subseteq U$ and that $\psi\left(u_{2}+\alpha(a)\right)=\psi\left(u_{2}\right)+\psi \alpha(a)=\psi\left(u_{2}\right)+g(a)=\psi(b)$. Thus $V_{2} \cap \operatorname{Im} \psi \subseteq \psi(U)$ and $\psi(U)$ has nonvoid interior in $\operatorname{Im} \psi$.

To establish the necessity, let $\psi: A \rightarrow X$ be a proper morphism in $\mathscr{L}$. Since $\operatorname{Im} \psi$ is necessarily closed in $X$, the natural projection $\beta$ of $X$ onto $X / \operatorname{Im} \psi$ is proper. Let $\alpha$ be the identity morphism from $A$ onto $A$. To see that (i) and (ii) are satisfied is elementary.

COROllary 2.2. Assume that

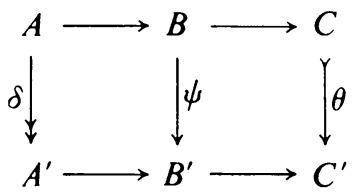

is a commutative diagram in $\mathscr{L}$ with proper exact rows. If $\delta$ is a proper epimorphism and $\theta$ is a proper monomorphism, then $\psi$ is proper.

Proposition 2.3. Let $\alpha: A \rightarrow X$ and $\beta: B \rightarrow X$ be morphisms in $\mathscr{L}$ and suppose that the diagram

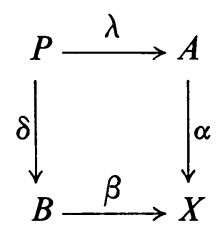

is a pullback for $\alpha$ and $\beta$. If $\alpha$ is proper, then so is $\delta$. The dual statement for pushouts is also true.

Proof. Let $M$ be the subgroup of $A \oplus B$ defined by $M=\{(a, b) \mid \alpha(a)=\beta(b)\}$. Then $M$ is closed in $A \oplus B$ and hence $M$ is in $\mathscr{L}$. The diagram

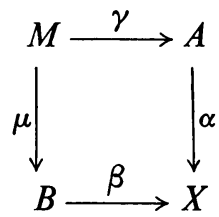

is a pullback for $\alpha$ and $\beta$ where $\mu:(a, b) \rightarrow b$ and $\gamma:(a, b) \rightarrow a$. Henceforth, we shall refer to this diagram as the standard pullback for $\alpha$ and $\beta$. Consider a neighborhood $W=(U \times V) \cap M$ of zero in $M$ where $U$ and $V$ are neighborhoods of zero in $A$ and $B$, respectively. Since $\alpha$ is proper, there is a neighborhood $E$ of $X$ such 
that $E \cap \operatorname{Im} \alpha=\alpha(U)$. Then $S=\beta^{-1}(E \cap \operatorname{Im} \beta) \cap V$ is a neighborhood of zero in $B$ and $\operatorname{Im} \mu \cap S \subseteq \mu(W)$. Thus $\mu$ is proper. If the diagram

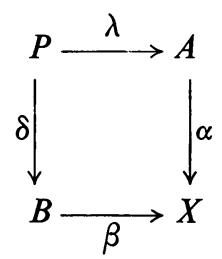

is also a pullback for $\alpha$ and $\beta$, we obtain a commutative diagram

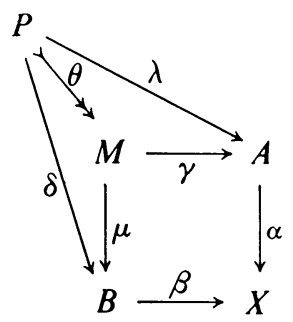

where $\theta$ is an isomorphism in $\mathscr{L}$. It easily follows from the commutativity of the above diagram that $\delta$ is proper. Pontrjagin duality yields the dual statement for pushouts.

Corollary 2.4. Let $A, B$ and $X$ be in $\mathscr{L}$. If $\alpha: A \rightarrow X$ and $\beta: B \rightarrow X$ are proper morphisms in $\mathscr{L}$, then every pullback for $\alpha$ and $\beta$ is a proper pullback. Dually, if $\gamma: X \rightarrow A$ and $\delta: X \rightarrow B$ are proper morphisms in $\mathscr{L}$, then every pushout for $\gamma$ and $\delta$ is a proper pushout.

We note that it is not always possible to construct pushouts in $\mathscr{L}$ in the "canonical fashion." In particular, the pushout for the pair of morphisms $\alpha, \beta$ : $R \rightarrow R / Z$ defined by $\alpha(x)=r_{1} x+Z$ and $\beta(x)=r_{2} x+Z$ for distinct fixed irrational numbers $r_{1}$ and $r_{2}$ may not be obtained in the standard way. However, when this construction is possible we shall refer to it as the standard pushout. The proof of the following proposition is straightforward and is omitted.

Proposition 2.5. If $\alpha: X \rightarrow A$ and $\beta: X \rightarrow B$ are morphisms in $\mathscr{L}$ and if $N=$ $\{(-\alpha(x), \beta(x)) \mid x \in X\}$ is closed in $A \oplus B$, then $\alpha$ and $\beta$ have a standard pushout. Moreover, if either $\alpha$ or $\beta$ is a proper monomorphism, then $N$ is closed in $A \oplus B$.

Proposition 2.6. Assume that

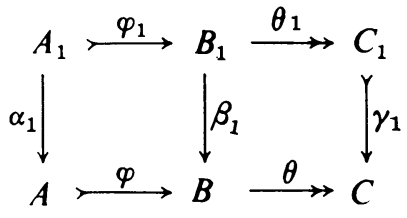

is a commutative diagram in $\mathscr{L}$ with proper short exact rows. If $\alpha_{1}$ is proper and $\gamma_{1}$ is a proper monomorphism, then $\beta_{1}$ is proper. 
Proof. Consider the commutative diagram

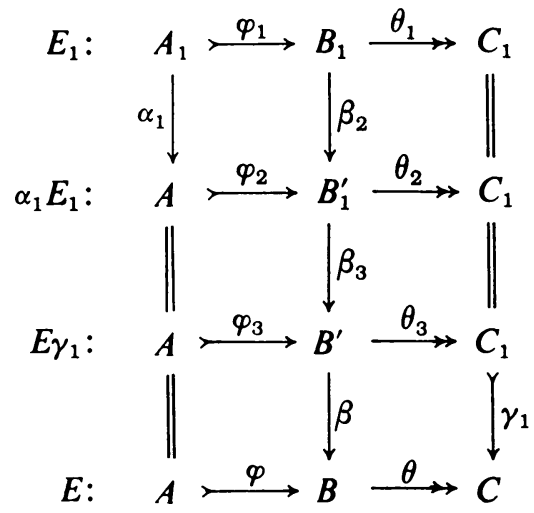

where $\alpha_{1} E_{1}$ is the standard pushout of $\varphi_{1}$ and $\alpha_{1}$ and $E \gamma_{1}$ is the standard pullback of $\theta$ and $\gamma_{1}$. Recall that $B_{1}^{\prime}=\left(A \oplus B_{1}\right) / N$ where $N=\left\{\left(-\alpha_{1}\left(a_{1}\right), \varphi_{1}\left(a_{1}\right)\right): a_{1} \in A_{1}\right\}$. Define $\beta_{3}: B_{1}^{\prime} \rightarrow B^{\prime}$ by

$$
\beta_{3}:\left(a, b_{1}\right)+N \rightarrow \varphi_{3}(a)+\left(\beta_{1}\left(b_{1}\right), \theta_{1}\left(b_{1}\right)\right) .
$$

The map $\beta_{3}$ is a continuous one-to-one, surjective homomorphism. By Corollary 2.2 , it follows that $\beta_{3}$ is an isomorphism of $\mathscr{L}$ and that $\beta$ is a proper monomorphism in $\mathscr{L}$. Since $\beta \beta_{3}$ is a proper monomorphism in $\mathscr{L}, \beta_{1}=\left(\beta \beta_{3}\right) \beta_{2}$ is a proper morphism in $\mathscr{L}$.

Applying Pontrjagin duality we obtain

Proposition 2.7. Assume that

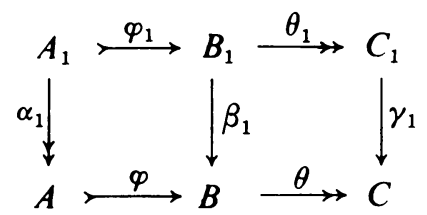

is a commutative diagram in $\mathscr{L}$ with proper short exact rows. If $\alpha_{1}$ is a proper epimorphism and $\gamma_{1}$ is proper, then $\beta_{1}$ is proper.

Our concluding result on commutative diagrams in $\mathscr{L}$ establishes a "Five Lemma" with respect to proper morphisms.

Proposition 2.8. Assume that the commutative diagram

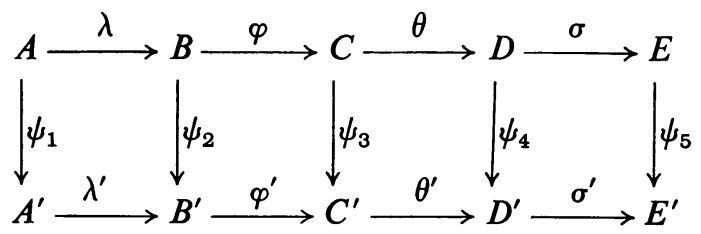


has proper exact rows. If $\psi_{1}, \psi_{2}, \psi_{4}$ and $\psi_{5}$ are proper isomorphisms, so is $\psi_{3}$. More precisely,

(i) if $\psi_{1}$ is a proper epimorphism and $\psi_{2}$ and $\psi_{4}$ are proper monomorphisms, then $\psi_{3}$ is a proper monomorphism.

(ii) if $\psi_{5}$ is a proper monomorphism and $\psi_{2}$ and $\psi_{4}$ are proper epimorphisms, then $\psi_{3}$ is a proper epimorphism.

Proof. Since (ii) can be obtained from (i) by Pontrjagin duality, it suffices to establish (i). Hence assume that $\psi_{1}$ is a proper epimorphism and that $\psi_{2}$ and $\psi_{4}$ are proper monomorphisms. We need only show that $\psi_{3}$ is proper. We first show that $\phi^{\prime} \psi_{2}$ is proper. Let $U$ be a neighborhood of zero in $B$. Since $\psi_{2}$ is proper, there is a neighborhood $V$ of $B^{\prime}$ such that $V \cap \operatorname{Im} \psi_{2}=\psi_{2}(U)$. Let $c^{\prime}$ be in the neighborhood $\phi^{\prime}(V) \cap \operatorname{Im} \phi^{\prime} \psi_{2}$ of $\operatorname{Im} \phi^{\prime} \psi_{2}$. Then there are elements $v \in V$ and $b \in B$ such that $c^{\prime}=\phi^{\prime}(v)=\phi^{\prime} \psi_{2}(b)$ which implies that $v-\psi_{2}(b) \in \operatorname{ker} \phi^{\prime}=\operatorname{Im} \lambda^{\prime}$. Since $\psi_{1}$ is surjective, we have that $\operatorname{Im} \lambda^{\prime} \subseteq \operatorname{Im} \psi_{2}$. Hence there is an element $b_{0} \in B$ such that $v-\psi_{2}(b)=\psi_{2}\left(b_{0}\right)$, that is, $v=\psi_{2}\left(b_{0}+b\right) \in V \cap \operatorname{Im} \psi_{2}=\psi_{2}(U)$. Since $\psi_{2}$ is a monomorphism, $b_{0}+b$ must be in $U$. Therefore, $c^{\prime} \in \phi^{\prime} \psi_{2}(U)$. Since clearly $\phi^{\prime}(V) \cap \operatorname{Im} \phi^{\prime} \psi_{2} \supseteq \phi^{\prime} \psi_{2}(U)$, it follows that $\phi^{\prime}(V) \cap \operatorname{Im} \phi^{\prime} \psi_{2}=\phi^{\prime} \psi_{2}(U)$. Thus $\phi^{\prime} \psi_{2}$ is proper. Note that $\psi_{4} \theta$ is a proper morphism since $\psi_{4}$ is monic. Thus $\psi_{3}$ is a morphism such that $\psi_{3} \phi$ and $\theta^{\prime} \psi_{3}$ are proper. Since also $\operatorname{Im} \phi=\operatorname{ker} \phi^{\prime} \psi_{3}$ we may apply the dual of Theorem 2.1 to obtain that $\psi_{3}$ is proper.

Moskowitz [10] has shown that $\mathscr{L}$ has neither enough projectives nor enough injectives. However, Moskowitz does show that if a group $G$ in $\mathscr{L}$ has a projective or injective resolution, then $G$ has a short projective or injective resolution, respectively. Our next result shows that one may approximate projective and injective resolutions for an arbitrary group in $\mathscr{L}$. These resolutions aid in certain computations of the Ext functor.

THEOREM 2.9. Let $G$ be a locally compact abelian group.

(1) If $K$ is a compact subgroup of $G$ such that $G / K$ has no small subgroups, then there exists a proper short exact sequence $G \longrightarrow I \oplus G / K \rightarrow J$ where $I$ and $J$ are compact injectives.

(2) Dually, if $K$ is a compactly generated open subgroup of $G$, then there exists a proper short exact sequence $P \succ K \oplus F \rightarrow G$ where $P$ and $F$ are (discrete) free projectives.

RemarK. Suppose $G$ is any group in $\mathscr{L}$. By Moskowitz's Theorem 2.7 [10], for each neighborhood $U$ of zero in $G$, there is a compact subgroup $K \subseteq U$ such that $G / K$ has no small subgroups. Thus the latter theorem applies to arbitrarily small subgroups $K$ of any $G$ in $\mathscr{L}$.

Proof of the theorem. Clearly it suffices to prove (1). Since $K$ is compact, $K$ has a short injective resolution $K_{\curlyvee}^{\lambda} I \rightarrow L$. We now obtain the commutative diagram where the second row is a proper pushout for $\lambda$ and $\delta$ and the morphism $\theta$ is the 


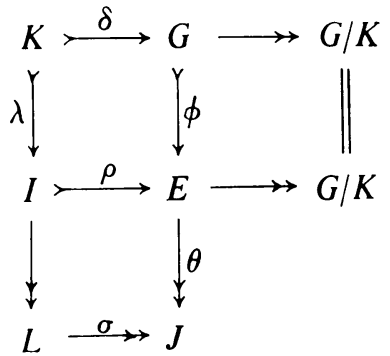

natural projection of $E$ onto $E / \operatorname{Im} \phi$. The morphism $\sigma$ is induced by $\rho$ and $\delta$. Since $L$ is compact, it follows that $\sigma$ is proper (see Hewitt and Ross [3]). Hence $J$ is a compact injective. Furthermore, since $I$ is injective, the second row splits and thus $E \cong I \oplus G / K$.

If $A$ and $C$ are in $\mathscr{L}$, then an extension of $A$ by $C$ is a proper short exact sequence $E: A \stackrel{\varphi}{\longrightarrow} B \stackrel{\theta}{\rightarrow} C$ where $\varphi$ and $\theta$ are morphisms in $\mathscr{L}$. If $E: A \stackrel{\varphi}{\longrightarrow} B \stackrel{\theta}{\rightarrow} C$ and $E^{\prime}: A \stackrel{\varphi^{\prime}}{\longrightarrow} B^{\prime} \stackrel{\theta^{\prime}}{\rightarrow} C^{\prime}$ are extensions of $A$ by $C$, then $E$ and $E^{\prime}$ are said to be congruent if and only if there is a morphism $\psi: B \rightarrow B^{\prime}$ in $\mathscr{L}$ such that the diagram

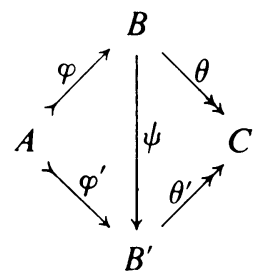

is commutative. It is a consequence of Corollary 2.2 , that $\psi$ is an isomorphism of $\mathscr{L}$. Denote by Ext $(C, A)$ the set of all congruence classes of extensions of $A$ by $C$. We intend to show that Ext is a functor from $\mathscr{L} \times \mathscr{L}$ into the category of discrete abelian groups which is contravariant in its first argument and covariant in the second. In view of the preceding results and Pontrjagin duality, it suffices to establish Lemma 1.3 of [7] since one may then follow Mac Lane's exposition to obtain the desired result.

Proposition 2.10. Assume that $\Gamma_{1}=\left(\alpha_{1}, \beta_{1}, \gamma_{1}\right): E_{1} \rightarrow E$ is a proper morphism from the extension $E_{1}: A_{1} \stackrel{\varphi_{1}}{\longrightarrow} B_{1} \stackrel{\theta_{1}}{\rightarrow} C_{1}$ to the extension $E: A \stackrel{\varphi}{\longrightarrow} B \stackrel{\theta}{\rightarrow} C$. Then $\Gamma_{1}$ has a unique factorization $E_{1} \stackrel{\left(\alpha_{1}, \beta^{\prime}, 1\right)}{\longrightarrow} E \gamma_{1} \stackrel{\left(1, \beta, \gamma_{1}\right)}{\longrightarrow} E$ where $\left(\alpha_{1}, \beta^{\prime}, 1\right)$ and $(1, \beta, \gamma)$ are proper morphisms.

Proof. Consider the commutative diagram of the proof of Proposition 2.6 (in the case we are considering here $\gamma_{1}$ is not necessarily monic). By Corollary 2.2, $\beta_{3}$ is an isomorphism in $\mathscr{L}$. By Corollary $2.4, \beta$ and $\beta_{2}$ are proper morphisms in $\mathscr{L}$. Since $\beta_{3}$ is an isomorphism, it follows that $\beta^{\prime}=\beta_{3} \beta_{2}$ is a proper morphism in $\mathscr{L}$. Thus $\left(\alpha_{1}, \beta^{\prime}, 1\right)$ and $\left(1, \beta_{1}, \gamma_{1}\right)$ are proper morphisms. The uniqueness follows from the uniqueness of proper pullbacks. 
For each extension $E$ of $A$ by $B$, let $[E]$ denote the class of all extensions $E^{\prime}$ of $A$ by $B$ such that $E^{\prime}$ is congruent to $E$.

Theorem 2.11. Assume that $A$ and $B$ are in $\mathscr{L}$. Then the class $\operatorname{Ext}(B, A)$ of all congruence classes of extensions of $A$ by $B$ is an abelian group with respect to the operation defined by

$$
\left[E_{1}\right]+\left[E_{2}\right]=\left[\nabla_{A}\left(E_{1} \oplus E_{2}\right) \Delta_{B}\right]
$$

where $E_{1}$ and $E_{2}$ are extensions of $A$ by $B$ and $\Delta_{B}$ and $\nabla_{A}$ are the diagonal and codiagonal morphisms, respectively. The correspondence Ext is an additive functor from $\mathscr{L} \times \mathscr{L}$ into the category of discrete abelian groups which is contravariant in the first variable and covariant in the second.

Proof. See Mac Lane [7, Chapter 3].

We now obtain further properties of the functor Ext. Observe that our functor Ext generalizes both the Ext functor as defined for discrete abelian groups and as defined by Moskowitz [10].

THeOrem 2.12. If $A$ and $B$ are in $\mathscr{L}$, then

$$
\operatorname{Ext}(A, B) \cong \operatorname{Ext}\left(B^{\wedge}, A^{\wedge}\right) .
$$

The isomorphism is natural.

Proof. Let $\psi: \operatorname{Ext}(A, B) \rightarrow \operatorname{Ext}\left(B^{\wedge}, A^{\wedge}\right)$ be the function induced by $E \rightarrow E^{\wedge}$ where $E=(B \stackrel{\varphi}{\longmapsto} X \stackrel{\theta}{\rightarrow} A)$ is an extension of $B$ by $A$ and $E^{\wedge}=\left(A^{\wedge} \stackrel{\theta^{\wedge}}{\longrightarrow} X^{\wedge} \stackrel{\varphi^{\wedge}}{\rightarrow} B^{\wedge}\right)$. The function $\psi$ is a natural isomorphism.

Let $\left\{G_{\lambda}\right\}_{\lambda \in \Lambda}$ be a family of groups in $\mathscr{L}$. We make $\bigoplus_{\lambda \in \Lambda} G_{\lambda}$ into a topological group by taking as a basis for the topology all sets of the form $\prod_{\lambda \in \Lambda} U_{\lambda} \cap \bigoplus_{\lambda \in \Lambda} G_{\lambda}$ where $U_{\lambda}$ is open in $G_{\lambda}$. If $\left\{G_{\lambda}\right\}_{\lambda \in \Lambda}$ is a family in $\mathscr{L}$, then $\prod_{\lambda} G_{\lambda}$ is in $\mathscr{L}$ if and only if $G_{\lambda}$ is compact for all but a finite number of $\lambda \in \Lambda$. Thus, if one wishes to topologize $\oplus_{\lambda} G_{\lambda}$ such that $\oplus_{\lambda} G_{\lambda}$ is in $\mathscr{L}$ and such that $\left(\oplus_{\lambda} G_{\lambda}\right)^{\wedge} \cong \Pi_{\lambda} G_{\lambda}^{\wedge}$, then $G_{\lambda}$ must be discrete for all but a finite number of $\lambda \in \Lambda$. Conversely, the topology defined on $\oplus_{\lambda} G_{\lambda}$ above yields that $\oplus_{\lambda} G_{\lambda}$ is in $\mathscr{L}$ and that $\left(\oplus_{\lambda} G_{\lambda}\right)^{\wedge} \cong \prod_{\lambda} G_{\lambda}$ whenever $G_{\lambda}$ is discrete for all but a finite number of $\lambda \in \Lambda$.

THEOREM 2.13. Assume that $\left\{G_{\lambda}\right\}_{\lambda \in \Lambda}$ is a family in $\mathscr{L}$ and that $H$ is in $\mathscr{L}$.

(1) If $\oplus_{\lambda} G_{\lambda}$ is in $\mathscr{L}$, then $\operatorname{Ext}\left(\oplus_{\lambda} G_{\lambda}, H\right) \cong \prod_{\lambda} \operatorname{Ext}\left(G_{\lambda}, H\right)$.

(2) If $\prod_{\lambda} G_{\lambda}$ is in $\mathscr{L}$, then $\operatorname{Ext}\left(H, \prod_{\lambda} G_{\lambda}\right) \cong \prod_{\lambda} \operatorname{Ext}\left(H, G_{\lambda}\right)$.

Proof. In view of Theorem 2.12 we need only establish (1): since Ext is an additive functor on $\mathscr{L} \times \mathscr{L}$, both (1) and (2) hold when $\Lambda$ is finite. Hence, recalling the discussion above, it suffices to assume that each $G_{\lambda}$ is discrete. Let $\pi_{\lambda}$ be the natural injection of $G_{\lambda}$ into $\oplus_{\lambda} G_{\lambda}$ and define $\sigma$ : Ext $\left(\oplus_{\lambda} G_{\lambda}, H\right) \rightarrow \prod_{\lambda}$ Ext $\left(G_{\lambda}, H\right)$ by $\sigma([E])=\left\langle\left[E \pi_{\lambda}\right]\right\rangle_{\lambda \in \Lambda}$. It is easily seen that $\sigma$ is a monomorphism. Let $\left\langle\left[E_{\lambda}\right]\right\rangle_{\lambda \in \Lambda}$ 
be in $\prod_{\lambda}$ Ext $\left(G_{\lambda}, H\right)$. Stripping $H$ of its topology, we form the extension $H \longmapsto$ $X \rightarrow \oplus_{\lambda} G_{\lambda}$ of discrete groups so that the diagram

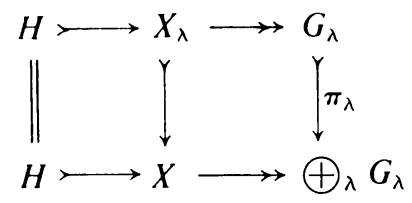

is commutative as a diagram of discrete abelian groups. We make $X$ into a topological group by taking as a base for its topology the collection of all sets of the form $x+U$ where $U$ is an open set in $H$ and $x \in X$. Let $E=\left(H \longmapsto X \rightarrow \oplus_{\lambda} G_{\lambda}\right)$. Clearly $\sigma([E])=\left\langle\left[E_{\lambda}\right]\right\rangle$.

Let $A \stackrel{\varphi}{\rightarrow} B \stackrel{\theta}{\rightarrow} C$ be a proper short exact sequence in $\mathscr{L}$, let $G$ be in $\mathscr{L}$ and let $f \in \operatorname{Hom}(A, G)$. Construct the pushout diagram for $f$ and $\varphi$

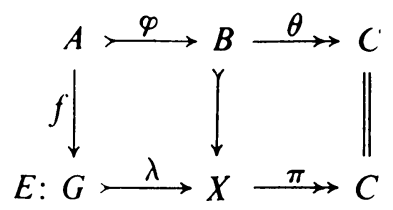

By Proposition 2.3, $\lambda$ is proper since $\varphi$ is proper. It is easily checked that $\pi$ is also proper. Thus, the homomorphism $\partial^{*}: \operatorname{Hom}(A, G) \rightarrow \operatorname{Ext}(C, G)$ may be defined in the usual way, i.e. $\partial(f)=[E]$. A similar argument holds for the homomorphism $\partial_{*}: \operatorname{Hom}(G, C) \rightarrow \operatorname{Ext}(G, A)$. The following theorem now follows from Proposition 2.10 and an argument analogous to the proof of Theorem 3.2 of [7].

THEOREM 2.14. Let $A \stackrel{\varphi}{\longrightarrow} B \stackrel{\theta}{\rightarrow} C$ be a proper short exact sequence in $\mathscr{L}$ and let $G$ be in $\mathscr{L}$. Then the two sequences

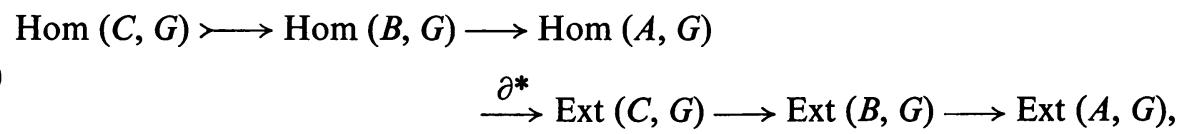

$\operatorname{Hom}(G, A) \succ \operatorname{Hom}(G, B) \longrightarrow \operatorname{Hom}(G, C)$

are exact.

REMARK. For each positive integer $n$, one can define a functor $\operatorname{Ext}^{n}$ from $\mathscr{L} \times \mathscr{L}$ into the category of discrete abelian groups. This functor is contravariant in its first argument and covariant in the second. Moreover, it reduces to the functor Ext we have already described in case $n=1$.

The functor $\mathrm{Ext}^{n}$ is obtained in a manner analogous to the corresponding development of the functor $\mathrm{Ext}^{n}$ in the theory of (discrete) abelian groups. We do not give the details of the construction, but instead refer the reader to the relevant 
chapters of Mac Lane [7]. It should be pointed out that modifications of Mac Lane's proofs must be made, but these modifications are minor in light of the homological algebra already developed in this paper. One consequence of this development is the following theorem:

THEOREM 2.15. Assume $G$ is a group in $\mathscr{L}$ and that $A \longmapsto B \rightarrow C$ is a proper exact sequence in $\mathscr{L}$. Then the sequence

$$
\begin{aligned}
\operatorname{Hom}(C, G) \longmapsto \operatorname{Hom}(B, G) \rightarrow & \operatorname{Hom}(A, G) \rightarrow \operatorname{Ext}(C, G) \rightarrow \operatorname{Ext}(B, G) \\
& \rightarrow \operatorname{Ext}(A, G) \rightarrow \operatorname{Ext}^{2}(C, G) \rightarrow \operatorname{Ext}^{2}(B, G) \rightarrow \cdots
\end{aligned}
$$

is exact. A dual result holds with $G$ in the first argument.

Proposition 2.16. (Notation is the same as that of 2.14.) If $C$ has a projective resolution or if $G$ has an injective resolution, then the sequence (1) is exact on the right end. Dually, if $A$ has an injective resolution or if $G$ has a projective resolution, then the sequence (2) is exact on the right end.

Proof. The duality theory already established makes it necessary only to prove that (1) is exact when either $C$ has a projective resolution or when $G$ has an injective resolution. From [10] if $C$ has a projective resolution, then $C$ has a short projective resolution. Likewise if $G$ has an injective resolution, then $G$ has a short injective resolution. Hence, in either case, a standard argument shows that $\operatorname{Ext}^{2}(C, G)=0$. Thus (1) is exact on the right end.

Proposition 2.17. Let $\operatorname{Ext}_{z}($, ) denote the extension functor on the category of discrete abelian groups and let $\mathscr{F}$ be the forgetful functor from $\mathscr{L}$ into the category of discrete abelian groups which forgets topological structure.

(a) If $A$ and $B$ are discrete, $\operatorname{Ext}(A, B) \cong \operatorname{Ext}_{z}(A, B)$.

(b) If $A$ and $B$ are compact, $\operatorname{Ext}(A, B) \cong \operatorname{Ext}_{z}\left(B^{\wedge}, A^{\wedge}\right)$.

(c) If $A$ is discrete and $B$ is in $\mathscr{L}$, $\operatorname{Ext}(A, B) \cong \operatorname{Ext}_{z}(A, \mathscr{F}(B))$.

(d) If $A$ is discrete and $B$ is divisible, Ext $(A, B)=0$.

(e) Suppose that $A$ is a totally disconnected group which contains a compact, bounded torsion subgroup $K$ such that $A / K$ is discrete. If $B$ is connected, then $\operatorname{Ext}(A, B)=0$.

(f) If $G$ is discrete, then $\operatorname{Ext}(T, G) \cong G$. Thus the range of $\operatorname{Ext}($,$) is all of the$ discrete abelian groups.

Proof. Both (a) and (b) are clear. If $A$ is discrete and if $B$ is in $\mathscr{L}$, then it is easily seen that there is a unique way of putting a topology on any extension group $X$ of $\mathscr{F}(B)$ by $A$ so that the sequence $B \succ X \rightarrow A$ is proper exact. This establishes (c). Clearly (d) follows from (c). Let $A$ satisfy the hypothesis of (e). By (d) we have that Ext $(A / K, B)=0$ since a connected group is divisible. Now $B=C \oplus R^{n}$ where $C$ is a compact connected group. Since $K^{\wedge}$ is a discrete, bounded torsion group and since $C^{\wedge}$ is torsion free and discrete, it follows that $0=\operatorname{Ext}\left(C^{\wedge}, K^{\wedge}\right)=$ 
Ext $(K, C)$. Hence Ext $(K, B)=0$ since $R^{n}$ is injective in $\mathscr{L}$. Thus, by exactness, Ext $(A, B)=0$. We remark that (e) generalizes a result of Braconnier [1]. Let $G$ be discrete. Then the exact sequence $Z \succ R \rightarrow T$ yields the exact sequence

$$
0=\operatorname{Hom}(R, G) \longrightarrow \operatorname{Hom}(Z, G) \stackrel{\partial}{\longrightarrow} \operatorname{Ext}(T, G) \longrightarrow \operatorname{Ext}(R, G)=0 .
$$

Hence $\operatorname{Hom}(Z, G) \cong \operatorname{Ext}(T, G)$. Since $G$ is discrete, $G \cong \operatorname{Hom}(Z, G)=\operatorname{Ext}(T, G)$.

3. The Second Noether Isomorphism Theorem in $\mathscr{L}$. It is well known that the Second Isomorphism Theorem does not hold in the category of abelian topological groups (for example, see [3]). However, it is an easy matter to see that it also fails for $\mathscr{L}$. Let $A$ denote the discrete group $R / Z$ and let $\varphi$ be a one-to-one, surjective homomorphism of $A$ onto $T$. Let $G=A \oplus T$ and let $B=\{(a, \varphi(a)): a \in A\}$. It is easily verified that $B$ is a closed discrete subgroup of $G$. Furthermore, $A \cap B=0$ and $G=A+B$. However $(A+B) / A=G / A=T$ and $B /(B \cap A)=B$. Before sharpening the version of the Second Isomorphism Theorem as given in [3], we prove the following technical lemma:

LEMMA 3.1. Let $G$ be in $\mathscr{L}$ and let $A$ and $B$ be closed subgroups of $G$ such that $G=A+B$ and $A \cap B=0$. Then $G=A \oplus B$ if and only if there is a closed subgroup $C \subseteq B$ such that the following two conditions hold:

(i) $A+C$ is closed in $G$.

(ii) The morphisms $\lambda: A \oplus C \rightarrow A+C$ and $\eta: B / C \rightarrow G /(A+C)$ are proper, where $\lambda:(a, c) \rightarrow a+c$ and $\eta: b+C \rightarrow b+(A+C)$.

Proof. The necessity is clear. Hence suppose that $C$ satisfies the hypothesis above and consider the commutative diagram

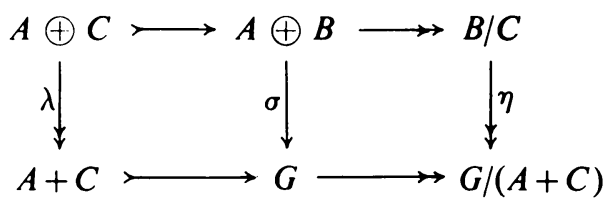

where $\sigma:(a, b) \rightarrow a+b$. By Theorem 2.1, $\sigma$ is proper. Thus $\sigma$ is an isomorphism in $\mathscr{L}$.

Corollary 3.2. Assume $G$ is in $\mathscr{L}$ and that $A$ and $B$ are closed subgroups of $G$ such that $G=A+B$ and $A \cap B=0$. If $B$ is $\sigma$-compact, then $G \cong A \oplus B$.

Proof. Apply Lemma 3.1 with $C=0$.

THEOREM 3.3. Let $G$ be in $\mathscr{L}$ and suppose that $A$ and $B$ are closed subgroups of $G$ such that $A+B$ is in $\mathscr{L}$. If $B$ is $\sigma$-compact, then

(i) $(A+B) / A \cong B /(A \cap B)$ and

(ii) $(A+B) / B \cong A /(A \cap B)$. 
Proof. The first isomorphism is just a restatement of Theorem 5.33 of [3]. Since $A+B$ is in $\mathscr{L}$, it follows that $A+B$ is closed in $G$. We also have that $(A+B) /(A \cap B)$ $=A /(A \cap B)+B /(A \cap B), \quad A /(A \cap B) \cap B /(A \cap B)=0$ and that $B /(A \cap B)$ is $\sigma$-compact. By Corollary 3.2, $(A+B) /(A \cap B)=A /(A \cap B) \oplus B /(A \cap B)$. Let $\theta$ be the natural homomorphism of $A+B$ onto $(A+B) /(A \cap B)$ and let $\pi$ be the natural projection of $(A+B) /(A \cap B)$ onto $A /(A \cap B)$. Then $\pi \theta$ is proper since $\pi$ is proper and $\theta$ is a proper epimorphism. Thus $(A+B) / B=(A+B) / \operatorname{ker} \pi \theta \cong A /(A \cap B)$.

We now establish two consequences of the above results which will be needed in the next section.

THEOREM 3.4. Let $D$ be a discrete group in $\mathscr{L}$. Then $\operatorname{Ext}(X, D)=0$ for all totally disconnected groups $X$ in $\mathscr{L}$ if and only if $D$ is divisible.

Proof. The necessity is clear. Therefore suppose that $D$ is a discrete divisible group and that $D \stackrel{i}{\rightarrow} A \rightarrow X$ represents an element of $\operatorname{Ext}(X, D)$. We may assume that $i$ is the inclusion homomorphism. It is elementary to see that $A$ is necessarily totally disconnected. Since $D$ is a discrete subgroup of $A$, there is a neighborhood $U$ of zero in $A$ such that $D \cap U=0$. By Theorem 2.7 of [10], there is a compact subgroup $K$ of $A$ contained in $U$ such that $A / K$ is discrete. The compactness of $K$ implies that $D+K$ is closed in $A$. Since $A / K$ is discrete, we have that $A / K=$ $(D+K) / K \oplus H / K$ for some subgroup $H$ of $A$. It follows that $D \cap H=0$ and that $A=D+H$. Let $\lambda: D \oplus K \rightarrow D+K$ and $\eta: H / K \rightarrow A /(D+K)$ be defined as in Lemma 3.1. By Corollary 3.2, $\lambda$ is an isomorphism. Since $A /(D+K) \cong$ $(A / K) /(D+K) / K$ is discrete, it follows that $\eta$ is proper. By Lemma 3.1, $A=D \oplus H$ and $D \stackrel{i}{\longrightarrow} A \rightarrow X$ splits.

THEOREM 3.5. Let $A \stackrel{\varphi}{\longrightarrow} G \stackrel{\theta}{\rightarrow} C$ be an extension of a discrete group $A$ by a connected group $C$. If $G_{0}$ is compact, then $G=G_{0} \oplus B$ where $B$ is discrete and $\theta\left(G_{0}\right)=C$.

Proof. Since $G_{0}$ is connected and since $G / G_{0}$ is totally disconnected, it is easily seen that $\varphi\left(G_{0}\right)=C$. Therefore $G=\varphi(A)+G_{0}$. Since $G_{0}$ is compact, we have by Theorem 3.3 (ii) that $G / G_{0}=\left(\varphi(A)+G_{0}\right) / G_{0} \cong \varphi(A) /\left(\varphi(A) \cap G_{0}\right)$. Therefore $G / G_{0}$ is discrete. By a theorem of Braconnier [1], $G=G_{0} \oplus B$ where $B$ is discrete.

4. Projectives and injectives in the category of totally disconnected, locally compact abelian groups. Let $\mathscr{D}$ denote the category of totally disconnected, locally compact abelian groups. We first remark that Moskowitz [10] has described the projectives and injectives in $\mathscr{L}$. In the next section we shall make use of the injectives for $\mathscr{L}$ and $\mathscr{D}$.

THEOREM 4.1. $P$ is a projective in $\mathscr{D}$ if and only if $P$ is a discrete free group.

Proof. By Theorem 3.3 of [10] the sufficiency is clear. Now suppose that $K$ is a compact projective in $\mathscr{D}$. Since $0=\operatorname{Ext}\left(K,(Q / Z)^{\wedge}\right) \cong \operatorname{Ext}_{z}\left(Q / Z, K^{\wedge}\right)$ where $Q$ denotes the discrete group of rational numbers, it follows that $K^{\wedge}$ is a discrete, 
divisible torsion group. If $K \neq 0$, then $K$ is a direct product of $p$-adic groups for various primes $p$. Viewed as an object of $\mathscr{L}, K$ is a projective relative to the compact groups in $\mathscr{L}$. If $K$ were also projective in $\mathscr{D}$, it would follow that $K$ is projective in $\mathscr{L}$. However, by Theorem 3.3 of [10], $K$ is not projective in $\mathscr{L}$ and hence not in $\mathscr{D}$. Thus $K=0$. Note, however, that a compact open subgroup of a projective of $\mathscr{D}$ is a projective of $\mathscr{D}$. This follows from the exact sequence alluded to in Proposition 2.16. Thus a projective $P$ in $\mathscr{D}$ has no nontrivial compact open subgroup and must necessarily be discrete. Therefore $P$ has a free resolution in $\mathscr{D}$ and thus $P$ is necessarily free.

THEOREM 4.2. $E$ is injective in $\mathscr{D}$ if and only if $E$ is a discrete divisible group.

Proof. The sufficiency follows from Theorem 3.4. Hence let $E$ be an injective in $\mathscr{D}$. Now $E$ contains a compact subgroup $K$ such that $E / K$ is discrete. We therefore obtain that

$$
\operatorname{Hom}(C, E / K) \rightarrow \operatorname{Ext}(C, K) \rightarrow \operatorname{Ext}(C, E)=0
$$

is exact for each compact totally disconnected $C$. Since $C$ is compact and $E / K$ is discrete, $\operatorname{Hom}(C, E / K)$ is torsion. Since $\operatorname{Ext}(C, K)$ is torsion, cotorsion, and reduced, Ext $(C, K)$ is bounded. If one chooses a basic subgroup $B$ of $K^{\wedge}$ one shows from the latter statement that $\operatorname{Ext}(B, J)$ is bounded for each discrete torsion $J$. From this one obtains that $B$ is bounded and that $K^{\wedge}=B \oplus D$ for divisible $D$. One may now argue that $D$ is zero and thus that each compact subgroup of $E$ is bounded. Now suppose that $K$ is infinite. Then there exists a prime $p$ and a positive integer $n$ such that $K^{\wedge}=\sum_{m} Z\left(p^{n}\right) \oplus L$ for some infinite cardinal $m$ and some subgroup $L$ of $K^{\wedge}$. Let $S=\sum_{m} Z\left(p^{n}\right) \oplus \sum_{k=1}^{\infty} \sum_{m} Z\left(p^{n k}\right)$. Then $p^{n} S=\sum_{k=2}^{\infty} \sum_{m} Z\left(p^{n k}\right)$ and $S / p^{n} S \cong \sum_{m} Z\left(p^{n}\right)$. Therefore, there is a morphism $\theta: E^{\wedge} \rightarrow S / p^{n} S$. Since $E^{\wedge}$ is necessarily projective relative to discrete torsion groups, there is a morphism $f: E^{\wedge} \rightarrow S$ such that the diagram

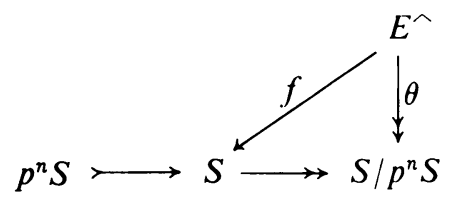

is commutative. Clearly $f$ is proper since $S$ is discrete and also $S=p^{n} S+H$ where $H=\operatorname{Im} f$. It is clear that $H$ is an unbounded direct sum of cyclic groups. By Pontrjagin duality, $E$ contains an unbounded compact subgroup which is a contradiction. Hence $K$ is finite and $E$ is discrete. Since $\operatorname{Ext}(Q / Z, E)=0$ where $Q$ denotes the discrete group of rational numbers, it follows that $E$ must be divisible.

5. The component of zero as a direct summand. We first remark that Proposition 2.17 (e) provides a sufficient condition on $G / G_{0}$ in order that $G_{0}$ be a direct summand of $G$. In this section we wish to find necessary and sufficient conditions on a connected group $C$ such that $C$ be a direct summand of every group containing 
$C$ as its component of zero. More generally we describe the structure of a group $E$ which has the property that $\operatorname{Ext}(X, E)=0$ for all totally disconnected groups $X$ in $\mathscr{L}$.

TheOREM 5.1. Let $C$ be a connected group in $\mathscr{L}$. Then Ext $(X, C)=0$ for all totally disconnected groups $X$ in $\mathscr{L}$ if and only if $C$ is an injective in $\mathscr{L}$.

Proof. The sufficiency is clear. Hence suppose $\operatorname{Ext}(X, C)=0$ for all totally disconnected groups $X$ in $\mathscr{L}$. Since $C$ is connected, $C=K \oplus R^{n}$ where $K$ is a compact connected group. It follows that $K^{\wedge}$ is torsion free and that $\operatorname{Ext}_{z}\left(K^{\wedge}, G\right)=0$ for all discrete torsion groups $G$. Hence $K^{\wedge}$ is a Baer-group which implies by Corollary 3.2 [2] that $K^{\wedge}$ is a free discrete group. Therefore $K=T^{\sigma}$ and $C=T^{\sigma} \oplus R^{n}$. By Theorem 3.2 of [10], $C$ is injective in $\mathscr{L}$.

TheOREM 5.2. Let $E$ be in $\mathscr{L}$. Then $\operatorname{Ext}(X, E)=0$ for all totally disconnected groups $X$ in $\mathscr{L}$ if and only if $E=I \oplus D$ where $I$ is injective in $\mathscr{L}$ and $D$ is a discrete divisible group.

Proof. Since $E_{0}$ is compactly generated, we have from [10] that $E_{0}$ has a short injective resolution $E_{0} \longrightarrow I \rightarrow J$. As in the proof of Theorem 2.9, this sequence induces the proper pushout diagram

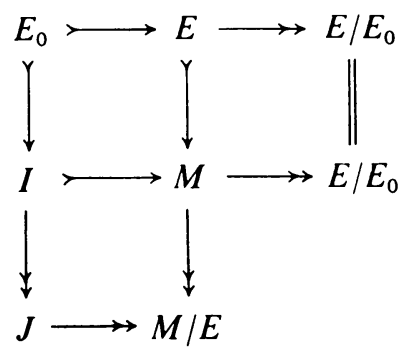

Since $I$ is injective, it follows that $M \cong I \oplus\left(E / E_{0}\right)$. Hence we obtain a proper exact sequence $E \longmapsto I \oplus\left(E / E_{0}\right) \rightarrow J$ where $I$ and $J$ are injective in $D$. Therefore, if $X$ is a totally disconnected group in $\mathscr{L}$, the exact sequence

$$
0=\operatorname{Ext}(X, E) \rightarrow \operatorname{Ext}\left(X, I \oplus\left(E / E_{0}\right)\right) \rightarrow \operatorname{Ext}(X, J)=0
$$

shows that $\operatorname{Ext}\left(X, E / E_{0}\right)=0$ for each $X$ in $\mathscr{D}$, that is, $E / E_{0}$ is injective in $\mathscr{D}$. Since $E / E_{0}$ is necessarily a discrete divisible group by Theorem 4.2, it follows by Proposition 2.17 (d) that $E=E_{0} \oplus D$ where $D$ is a discrete divisible group. Theorem 5.1 yields that $E_{0}$ is injective in $\mathscr{L}$ which completes the proof.

\section{REFERENCES}

1. Jean Braconnier, Sur les groupes topologiques localement compacts, J. Math. Pures Appl. (9) 27 (1948), 1-85. MR 10, 11.

2. Phillip A. Griffith, A solution to the splitting mixed group problem of Baer, Trans. Amer. Math. Soc. 139 (1969), 261-269. 
3. Edwin Hewitt and Kenneth Ross, Abstract harmonic analysis. Vol. I: Structure of topological groups. Integration theory, group representations, Die Grundlehren der math. Wissenschaften, Band 115, Academic Press, New York, and Springer-Verlag, Berlin, 1963. MR 28 \#158.

4. G. Hochschild, Group extensions of Lie groups. I, Ann. of Math. (2) 54 (1951), 96-109. MR 13, 12.

5. - Group extensions of Lie groups. II, Ann. of Math. (2) 54 (1951), 537-551. MR 13, 318.

6. K. H. Hofmann and P. S. Mostert, Splitting in topological groups, Mem. Amer. Math. Soc. No. 43 (1963). MR 27 \#1529.

7. Saunders Mac Lane, Homology, Die Grundlehren der math. Wissenschaften, Band 114, Academic Press, New York, and Springer-Verlag, Berlin, 1963. MR 28 \#122.

8. Calvin C. Moore, Extensions and low dimensional cohomology theory of locally compact groups. I, Trans. Amer. Math. Soc. 113 (1964), 40-63. MR 30 \#2106.

9. - Extensions and low dimensional cohomology theory of locally compact groups. II, Trans. Amer. Math. Soc. 113 (1964), 64-86. MR 30 \#2106.

10. Martin Moskowitz, Homological algebra in locally compact abelian groups, Trans. Amer. Math. Soc. 127 (1967), 361-404. MR 35 \#5861.

11. L. S. Pontrjagin, Topological groups, GITTL, Moscow, 1954; English transl., Gordon and Breach, New York, 1966. MR 17, 171; MR 34 \#1439.

UNIVERSITY OF HOUSTON, Houston, TEXAS 77004 